\title{
Straumann juggernaut launches national UK digital tour
}

Straumann UK invites you to rock out this summer at one of the most instrumental roadshows UK dentistry has ever seen. They will have you jamming to the tune of digital success as they unveil a number of exciting developments to their digital solutions portfolio.

For ten days in July the 'Straumann Juggernaut' will play host to the Digital Performance tour, taking to the road and visiting seven locations across the UK. This highly-anticipated rock fest will showcase to clinicians, technicians and CDTs the potential to amplify their career with the NEW synchronised digital workflow from Straumann CARES Digital Solutions.

This epic roadshow is a result of Straumann's commitment to ensure clinicians, technicians and CDTs have everything they need for every step of the treatment pathway. Audiences can expect to see exclusive performances from headlines acts such as Case Planning \& Guided Surgery, Digital Impressions, Lab Scanners, In-Lab \& Centralised Milling, 3D Printing and Scan \& Shape.

\section{ROCK OUT TO DIGITAL...}

The new and exciting Straumann CARES Digital Solutions offers the complete digital workflow to help achieve precision and efficiency, together with the peace of mind of Straumann's quality, reliability and service. Combining interconnected software platforms, open and fully validated workflows alongside a wide variety of materials on offer - Straumann CARES Digital Solutions is a true benchmark in digital dentistry.

\section{TOUR DATES}

Audiences will be driven wild by the great product deals, insightful presentations and hands-on demonstrations on offer at the Straumann Digital Performance Tour. Plus, with exclusive backstage access to the live music and entertainment on offer, and after parties scheduled at selected locations, the Straumann Digital Performance Tour promises to be a must-attend event for any technician, $\mathrm{CDT}$ or clinician looking to enhance their digital offering in 2017 and beyond.

- 10-11 JULY LONDON

- 12 JULY EXETER

- 13-14 JULY COVENTRY

- 17-18 JULY MANCHESTER

- 19 JULY LEEDS

- 20 JULY NEWCASTLE

- 21 JULY EDINBURGH

Don't miss the opportunity to see these fantastic innovations at the Straumann Digital Performance Tour. FREE to attend places are limited - to book your place visit straumanndigitalperformance.co.uk or call 01293651230 .

straumanndigitalperformance.co.uk

straumann.co.uk

therevu.co.uk

Facebook: Straumann UK

Twitter: @StraumannUK

\#digitalperformancetour

\section{CASE PLANNING \& GUIDED}

SURGERY - The workflow between coDiagnostiX and Straumann CARES Visual is completely seamless with DWOS Synergy. Transfer coDiagnostiX implant planning to CARES Visual and receive the restorative plan from the lab technician. Provides complete data visualisation to achieve real-time surgical and restorative case planning.

INTRA-ORAL SCANNING - Designed for high performance, ease of use and optimal patient comfort, the extremely compact Straumann CARES IO Scanner and Straumann CARES IO Portable Scanner allows clinicians to quickly and easily create digital impression data that can be used to design and produce effective
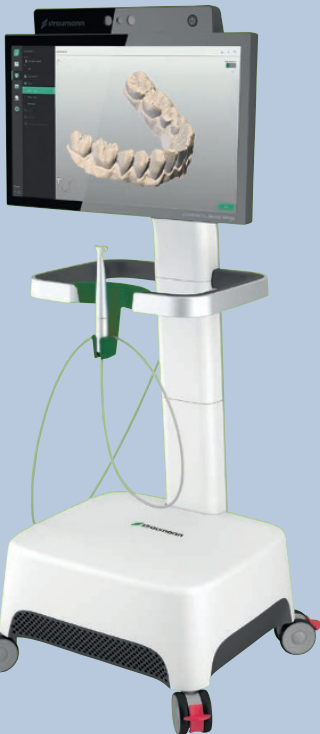

Straumann IO Scanner

prosthodontic solutions. 3Shape TRIOS Wireless Intraoral Scanners enhances patient experience, reduces chair-time and unlocks the widest range of treatment opportunities. Backed by continuous software updates, upgradable hardware and add-on treatment modules, the 3Shape TRIOS 3 range of intraoral scanners offers accurate, ultra-fast, powder-free scanning.

LAB SCANNERS - Straumann CARES 3Series and 7Series Desktop Scanners effectively combine proven and established scanning process with the latest computer technology in an elegant, functional design, to accurately scan models and impressions. The scanners combine proven laser triangulation technology with three or five axes of freedom and are embedded with a powerful PC and several DWOS applications.

IN-HOUSE MILLING \& 3D PRINTING - Straumann offers the capability of milling custom dental prosthetics in-house via the flexible Straumann CARES Series Milling Units (C, D \& M Series). Additionally, the Straumann CARES P Series 3D Printer sets a new standard in 3D printing for labs, offering speed and reliability specially tailored to satisfy the highest demands in both the practice and laboratory.

\section{OUTSOURCED PRODUCTION - The Straumann CARES}

Centralised Milling facility operates as an extension of the modern laboratory, with state-of-the art equipment and validated workflows. There is a comprehensive range of products to choose from, including one-step restorations, customised abutments, screw-retained bridges and bars, copings, bridge frameworks as well as full contour crowns and bridges. Alongside this, Straumann offer a variety of materials including multiple glass ceramics, zirconia available in numerous shades and translucencies, cobalt chrome, titanium and polymers.

By adding an all-encompassing digital dimension to their portfolio Straumann have added a completely new vibe to their offering - they're more than a dental implant company, they're your digital solutions partner. 